PROCEEDINGS OF THE

AMERICAN MATHEMATICAL SOCIETY

Volume 34, Number 2, August 1972

\title{
TORSION THEORIES OVER SEMIPERFECT RINGS
}

EDGAR A. RUTTER, JR.

\begin{abstract}
In this note we characterize those torsion theories over a semiperfect ring such that the class of torsion free modules is closed under projective covers and the hereditary torsion theories for which this is true of the class of torsion modules. These results are applied to determine all hereditary torsion theories over an injective cogenerator ring with the property that the torsion submodule of every module is a direct summand.
\end{abstract}

The purpose of this note is to investigate some properties of torsion theories in the category of modules over a semiperfect ring. We characterize those torsion theories with the property that the class of torsion free modules is closed under projective covers and the hereditary torsion theories for which this is true of the class of torsion modules. A torsion theory is called splitting provided the torsion submodule of every module is a direct summand. Bernhardt [4] proved that every splitting hereditary torsion theory for a quasi-Frobenius ring is determined by a central idempotent of the ring. The results mentioned above permit us to extend this result to a natural generalization of quasi-Frobenius rings, namely, the one-sided injective cogenerator rings. Bernhardt's theorem depended on a result of Alin and Armendariz [1] which states that every hereditary torsion class over a right perfect ring is closed under direct products and hence is determined by an idempotent ideal of the ring. While we shall not require this result our methods yield a new proof of it.

Throughout this paper the term "ring" will mean an associative ring with identity and all modules will be unital left modules. We denote the category of all modules over a ring $R$ by ${ }_{R} \mathscr{M}$. Dickson [6] defined a torsion theory for ${ }_{R} \mathscr{M}$ to be a pair $(\mathscr{T}, \mathscr{F})$ of classes of modules satisfying:

(a) $\mathscr{T} \cap \mathscr{F}=\{0\}$;

(b) $\mathscr{T}$ is closed under homomorphic images and $\mathscr{F}$ is closed under submodules;

(c) for each module $M$ there exists a unique submodule $M_{t}$ of $M$ such that $M_{t} \in \mathscr{T}$ and $M / M_{t} \in \mathscr{F}$.

Presented to the Society, January 18, 1972; received by the editors March 15, 1971. AMS 1969 subject classifications. Primary 1640, 1650.

Key words and phrases. Hereditary torsion theory, splitting torsion theory, torsiontorsion free class, injective hull, projective cover, semiperfect ring, injective cogenerator ring.

(C) American Mathematical Society 1972 
A class of modules $\mathscr{T}$ is a torsion class if there exists a class $\mathscr{F}$ such that $(\mathscr{T}, \mathscr{F})$ is a torsion theory. A torsion class $\mathscr{T}$ is closed under homomorphic images, direct sums, and extensions and uniquely determines $\mathscr{F}$. Conversely, any class of modules with these three properties is a torsion class. A torsion free class is defined dually and is characterized by being closed under submodules, direct products, and extensions. A torsion class $\mathscr{T}$ and the associated torsion theory $(\mathscr{T}, \mathscr{F})$ is called hereditary if $\mathscr{T}$ is closed under submodules. This is equivalent to $\mathscr{F}$ being closed under injective envelopes. All of the above information is contained in [6].

A ring $R$ is semiperfect if each cyclic $R$-module has a projective cover. This implies that each finitely generated $R$-module has a projective cover. For the basic properties of semiperfect rings see [3] or [9].

Proposition 1. Let $(\mathscr{T}, \mathscr{F})$ be a torsion theory in ${ }_{R} \mathscr{M}$. If $\mathscr{F}$ is closed under homomorphic images, then $\mathscr{T}$ is closed under minimal epimorphisms. Conversely, suppose that $(\mathscr{T}, \mathscr{F})$ is hereditary and $R$ is semiperfect. Then if $\mathscr{T}$ is closed under projective covers of simple modules, $\mathscr{F}$ is closed under homomorphic images.

Proof. Let $M^{\prime} \in \mathscr{T}$ and $M \rightarrow M^{\prime} \rightarrow 0$ be a minimal epimorphism with kernel $K$. Then

$$
M /\left(K+M_{t}\right) \cong(M / K) /\left(\left(K+M_{t}\right) / K\right) \in \mathscr{T}
$$

since $M^{\prime} \in \mathscr{T}$. However, $M / M_{t} \in \mathscr{F}$ and so

$$
M /\left(K+M_{t}\right) \cong\left(M / M_{t}\right) /\left(\left(K+M_{t}\right) / M_{t}\right) \in \mathscr{F}
$$

by hypothesis. Since $\mathscr{T} \cap \mathscr{F}=\{0\}, M=K+M_{t}$ and so $M=M_{t} \in \mathscr{T}$.

Suppose $\mathscr{T}$ is closed under projective covers of simple modules. Since $\mathscr{T}$ is closed under homomorphic images, for any finitely generated module $M \in \mathscr{T}$, the projective cover of $M, P(M) \in \mathscr{T}$. Let $W \rightarrow W^{\prime} \rightarrow 0$ be an epimorphism with $W \in \mathscr{F}$. Since $\operatorname{Hom}(P(M), W)=0$, the exactness of the sequence

$$
\operatorname{Hom}(P(M), W) \rightarrow \operatorname{Hom}\left(P(M), W^{\prime}\right) \rightarrow 0
$$

implies $\operatorname{Hom}\left(P(M), W^{\prime}\right)=0$. Therefore, the exactness of the sequence

$$
0 \rightarrow \operatorname{Hom}\left(M, W^{\prime}\right) \rightarrow \operatorname{Hom}\left(P(M), W^{\prime}\right)
$$

implies $\operatorname{Hom}\left(M, W^{\prime}\right)=0$. Since $\mathscr{T}$ is closed under submodules and $M$ is an arbitrary finitely generated module in $\mathscr{T}, W_{t}^{\prime}=0$ and so $W^{\prime} \in \mathscr{F}$.

In [8] Jans called a hereditary torsion class $\mathscr{T}$ a torsion-torsion free (TTF) class provided $\mathscr{T}$ is closed under direct products. By virtue of results of Dickson [6] cited earlier, $\mathscr{T}$ is a TTF class if and only if there exist torsion theories $(\mathscr{T}, \mathscr{F})$ and $(\mathscr{C}, \mathscr{T})$ in ${ }_{R} \mathscr{M}$. It is shown in [8] that 
there exists a one-to-one correspondence between TTF classes in ${ }_{R} \mathscr{M}$ and idempotent ideals of $R$. The correspondence is given as follows: $I=R_{c}$, the $\mathscr{C}$-torsion submodule of $R$, while $\mathscr{T}_{I}=\left\{M\right.$ in $\left.{ }_{R} \mathscr{M} \mid I M=0\right\}$.

If $P$ is a projective $R$-module, $T=\sum_{f} \operatorname{Im}(f)$ where $f \in \operatorname{Hom}(P, R)$ is an ideal of $R$ called the trace ideal of $P$. It is immediate from the "dual basis lemma" [5, VII, 3.1] that $T^{2}=T$ and $T P=P$.

Proposition 2. Let $R$ be a semiperfect ring and $(\mathscr{T}, \mathscr{F})$ be a hereditary torsion theory in ${ }_{R} \mathscr{M}$. If $\mathscr{F}$ is closed under homomorphic images, there exists an idempotent ideal $T$ of $R$ such that $\mathscr{T}=\mathscr{T}_{T}$. In particular $\mathscr{T}$ is a TTF class.

Proof. Let $S_{1}, \cdots, S_{k}$ be a complete set of representatives for the isomorphism classes of simple $R$-modules in $\mathscr{F}, P$ and $Q$ denote, respectively, the projective cover and injective hull of $S_{1} \oplus \cdots \oplus S_{k}$, and $T$ be the trace ideal of $P$. We show first that $\mathscr{F}$ consists precisely of those modules which can be embedded in a direct product of copies of $Q$. We denote the class of such modules by $\mathscr{S}$. Since $(\mathscr{T}, \mathscr{F})$ is hereditary and $S_{1} \oplus \cdots \oplus S_{k} \in \mathscr{F}, Q \in \mathscr{F}$ and so $\mathscr{S} \subseteq \mathscr{F}$. If $X \in \mathscr{F}$, then for any $0 \neq x \in X, R x$ has a simple epimorphic image which is contained in $Q$. Since $Q$ is injective, there exists $f \in \operatorname{Hom}(X, Q)$ such that $f(x) \neq 0$. Thus $X \in \mathscr{S}$ and hence $\mathscr{F}=\mathscr{S}$.

The unique torsion free class determined by $\mathscr{T}_{T}$ is

$$
\mathscr{F}_{T}=\left\{M \text { in }_{R} \mathscr{M} \mid\{m \in M \mid T m=0\}=0\right\} .
$$

Hence $Q \in \mathscr{F}_{T}$ since $S_{1} \oplus \cdots \oplus S_{k}$ does and $\left(\mathscr{T}_{T}, \mathscr{F}_{T}\right)$ is hereditary. Thus $\mathscr{F}_{\mathscr{F}} \subseteq \mathscr{F}_{T}$ and so $\mathscr{T}_{T} \subseteq \mathscr{T}$. Suppose $M \in \mathscr{T}$, then $\operatorname{Hom}(P, M)=0$. For if $0 \neq f \in \operatorname{Hom}(P, M), f(P)$, being a homomorphic image of $P$ which is finitely generated, has a simple homomorphic image which is also a homomorphic image of $P$ and so isomorphic to a submodule of $Q$. This implies $\operatorname{Hom}(f(P), Q) \neq 0$ and hence $\operatorname{Hom}(M, Q) \neq 0$ since $Q$ is injective. This contradiction shows $\operatorname{Hom}(P, M)=0$ and so $M \in \mathscr{T}_{T}$. Thus $\mathscr{T}=\mathscr{T}_{T}$.

THEOREM 3. If $R$ is a right perfect ring and $(\mathscr{T}, \mathscr{F})$ is a hereditary torsion theory in $R^{\mathscr{M}}$, there exists an idempotent ideal $T$ of $R$ such that $(\mathscr{T}, \mathscr{F})=\left(\mathscr{T}_{T}, \mathscr{F}_{T}\right)$. In particular, $\mathscr{T}$ is a TTF class.

The only modification needed in proof of Proposition 2 to establish this result is to observe that since $R$ is right perfect each $X \in \mathscr{F}$ has an essential socle which belongs to $\mathscr{F}$ and so $X \in \mathscr{S}$. The proof of this proposition exhibits a one-to-one correspondence between hereditary torsion classes in ${ }_{R} \mathscr{M}$ and sets of isomorphism classes of simple left $R$-modules. Thus if $R$ has $n$ nonisomorphic simple left modules, there are $2^{n}$ hereditary torsion classes in ${ }_{R} \mathscr{M}$ and each is a TTF class. Similar 
classifications of the hereditary torsion theories in ${ }_{R} \mathscr{M}$ were obtained earlier by Alin and Armendariz [1] by extending a method of Jans [8] and by Dlab [7]. In each instance the approach differs from that above. Finally we note that the above results show that every idempotent ideal in a left or right perfect ring is the trace ideal of a finitely generated projective module.

THEOREM 4. Let $R$ be a semiperfect ring and $(\mathscr{T}, \mathscr{F})$ be a torsion theory in ${ }_{R} \mathscr{M}$. Then the following conditions are equivalent:

(a) $P_{t}$ is a direct summand of $P$ for every projective module $P$ in ${ }_{R} \mathscr{M}$;

(b) $\mathscr{F}$ is closed under projective covers;

(c) $\mathscr{F}$ is closed under projective covers of cyclic modules;

(d) $R_{t}$ is a direct summand of ${ }_{R} R$.

Proof. (a) $\Rightarrow(\mathrm{b})$. Let $X \in \mathscr{F}$ and $P \rightarrow X$ be a projective cover with kernel $K$. Since $P_{t} \subseteq K$ and $P_{t}$ is a summand of $P$, we have $P_{t}=0$. Thus $P \in \mathscr{F}$.

(b) $\Rightarrow$ (c). This implication is trivial.

(c) $\Rightarrow\left(\right.$ d). Let $\pi: P \rightarrow R / R_{t}$ be a projective cover. There exists an epimorphism $\alpha: R \rightarrow P$ such that $\pi \cdot \alpha=\eta$, the natural projection of $R$ on $R / R_{t}$. Since $P$ is projective $R=\operatorname{Ker} \alpha \oplus P^{\prime}$ with $P^{\prime}$ isomorphic to $P$. Thus

$$
R_{t}=(\operatorname{Ker} \alpha)_{t} \oplus P_{t}^{\prime}=(\operatorname{Ker} \alpha)_{t} \quad \text { as } \quad P^{\prime} \in \mathscr{F} .
$$

However, $\pi \cdot \alpha=\eta$ implies Ker $\alpha \subseteq R_{t}$. Thus $R_{t}=\operatorname{Ker} \alpha$ and so is a direct summand of ${ }_{R} R$.

(d) $\Rightarrow$ (a). If $P$ is a projective $R$-module there exists an $R$-module $Q$ such that $F=P \oplus Q$ is a free module. It is immediate from (d) that $F_{t}$ is a direct summand of $F$. Since $F_{t}=P_{t} \oplus Q_{t}$, it follows that $P_{t}$ is a direct summand of $F$ and hence also of $P$.

THEOREM 5. Let $R$ be a semiperfect ring and $(\mathscr{T}, \mathscr{F})$ be a hereditary torsion theory in ${ }_{R} \mathscr{M}$. Then the following conditions are equivalent:

(a) $\mathscr{F}$ is closed under homomorphic images;

(b) $\mathscr{T}$ is closed under projective covers;

(c) $\mathscr{T}$ is closed under projective covers of simple modules;

(d) $(\mathscr{T}, \mathscr{F})=\left(\mathscr{T}_{T}, \mathscr{F}_{T}\right)$ where $T=R e$ is an ideal of $R$ and $e$ an idempotent element of $R$.

Proof. The equivalence of conditions (a)-(c) is immediate from Proposition 1. Assume (a)-(c) hold. Then it follows from Proposition 2 that $(\mathscr{T}, \mathscr{F})=\left(\mathscr{T}_{T}, \mathscr{F}_{T}\right)$ where $T$ is an idempotent ideal of $R$. Furthermore, $\left(\mathscr{C}_{T}, \mathscr{T}_{T}\right)$ is a torsion theory in ${ }_{R} \mathscr{M}$ where $\mathscr{C}_{T}=\left\{Y \in_{R} \mathscr{M} \mid T Y=Y\right\}$. It follows from Theorem 4 applied to this torsion theory that $R_{c}=T$ is a direct summand of ${ }_{R} R$ and so $T=R e$ with $e$ an idempotent element of $R$. 
The implication $(\mathrm{d}) \Rightarrow$ (c) also follows from Theorem 4 applied to the torsion theory $\left(\mathscr{C}_{T}, \mathscr{T}_{T}\right)$.

A torsion theory $(\mathscr{T}, \mathscr{F})$ in ${ }_{R} \mathscr{M}$ is centrally splitting if $\mathscr{T}$ is a TTF class and the associated idempotent ideal is a ring direct summand of $R$, that is, it is principal generated by a central idempotent of $R$. Bernhardt [4] called a torsion theory for a semiperfect ring principal provided, for every simple module $S$ in ${ }_{R} \mathscr{M}$,

(i) $S$ is in $\mathscr{T}$ if and only if the projective cover of $S$ is in $\mathscr{T}$, and

(ii) $S$ is in $\mathscr{F}$ if and only if the projective cover of $S$ is in $\mathscr{F}$.

THEOREM 6. Let $R$ be a semiperfect ring and $(\mathscr{T}, \mathscr{F})$ be a hereditary torsion theory in ${ }_{R} \mathscr{M}$. Then the following conditions are equivalent:

(a) $\mathscr{T}$ is closed under injective hulls and projective covers of simple modules;

(b) $(\mathscr{T}, \mathscr{F})$ is principal;

(c) $(\mathscr{T}, \mathscr{F})$ is centrally splitting.

Proof. (a) $\Rightarrow$ (c). It follows from Theorem 5 that there exists an ideal $T=R e$ with $e^{2}=e \in R$ such that $(\mathscr{T}, \mathscr{F})=\left(\mathscr{T}_{T}, \mathscr{F}_{T}\right)$. Thus $\mathscr{T}$ is a TTF class. Let $S_{1}, \cdots, S_{k}$ be distinct representatives for the isomorphism classes of simple modules in $\mathscr{T}$. Then if $Q$ is the injective hull of

$$
S_{1} \oplus \cdots \oplus S_{k}
$$

it follows exactly as in the proof of Proposition 2 that $\mathscr{T}$ is the class of modules in ${ }_{R} \mathscr{M}$ cogenerated by $Q$ and so is closed under injective envelopes. Hence the torsion class $\mathscr{C}_{T}$ is hereditary. Thus for each $x \in T$, $R x=T R x=T x$, that is, $x \in T x$. We complete the proof of this implication by showing that $T=f R$ with $f^{2}=f$. For this it suffices to show that if $f_{i}$ is a primitive idempotent of $R, f_{i} T \neq 0$ implies $f_{i} T=f_{i} R$. If not, then since $f_{i} J$ is the unique maximal submodule of $f_{i} R$ where $J$ is the Jacobson radical of $R, f_{i} T \subseteq f_{i} J$. Then for each $x \in f_{i} T$ there is a $t \in T$ such that $t x=x$. Thus $f_{i} t x=f_{i} x=x$ and so $\left(1-f_{i} t\right) x=0$. Since $f_{i} t \in f_{i} J \subseteq J, x=0$. This contradiction completes the proof of the implication.

(c) $\Rightarrow$ (a). This implication is clear.

(b) $\Leftrightarrow$ (c). This equivalence was proved by Bernhardt [4] under the added hypothesis that $\mathscr{T}$ is a TTF class. However, if $(\mathscr{T}, \mathscr{F})$ is principal it follows from Theorem 5 that $\mathscr{T}$ is a TTF class. The rest of the proof follows exactly as in [4].

If $(\mathscr{T}, \mathscr{F})$ is a torsion theory for ${ }_{R} \mathscr{M}$ and $M \in R_{R} \mathscr{M}$, then $M$ is said to split provided $M=M_{t} \oplus M^{\prime}$ and $(\mathscr{T}, \mathscr{F})$ is called a splitting torsion theory if every module in $R^{\mathscr{M}}$ splits. A centrally splitting torsion theory is always a splitting torsion theory. In fact a TTF class $\mathscr{T}$ with associated torsion 
theories $(\mathscr{C}, \mathscr{T})$ and $(\mathscr{T}, \mathscr{F})$ is centrally splitting if and only if

$$
M=M_{t} \oplus M_{c}
$$

for every $M$ in ${ }_{R} \mathscr{M}$ (see [8]).

A ring $R$ is a (left) injective cogenerator ring if ${ }_{R} R$ is an injective cogenerator in ${ }_{R} \mathscr{M}$. These rings were first studied by Osofsky [10]. This class of rings has also arisen in a somewhat different fashion in the work of Azumaya [2] and Utumi [11].

THEOREM 7. Let $R$ be a left or right injective cogenerator ring and $(\mathscr{T}, \mathscr{F})$ be a hereditary torsion theory in ${ }_{R} \mathscr{M}$. Then the following conditions are equivalent:

(a) $\mathscr{T}$ is closed under injective hulls of simple modules;

(b) $\mathscr{T}$ is closed under projective covers of simple modules;

(c) $(\mathscr{T}, \mathscr{F})$ is principal;

(d) $(\mathscr{T}, \mathscr{F})$ is splitting;

(e) $(\mathscr{T}, \mathscr{F})$ is centrally splitting.

Proof. Osofsky [10] showed $R$ is semiperfect. If $e_{1}, \cdots, e_{n}$ is a complete set of primitive orthogonal idempotents for $R$ it follows from [11] that each $R e_{i}$ contains a unique simple submodule $S_{i}$ and a unique simple factor module $S_{i}^{\prime}$ for which it is an essential extension and a projective cover, respectively. Moreover, as $i$ ranges from 1 to $n$ the $S_{i}$ and $S_{i}^{\prime}$ provide distinct representatives for the isomorphism classes of simple $R$-modules. We may assume they are indexed so that $S_{1}, \cdots, S_{k} \in \mathscr{T}$ and $S_{k+1}, \cdots, S_{n} \in \mathscr{F}$ and since $(\mathscr{T}, \mathscr{F})$ is hereditary, $R e_{k+1}, \cdots, R e_{n} \in \mathscr{F}$.

If (a) holds, $R e_{1}, \cdots, R e_{k} \in \mathscr{T}$. Since no module in $\mathscr{F}$ has a projective cover in $\mathscr{T}$ as $\operatorname{Hom}(M, X)=0$ for all $M \in \mathscr{T}$ and $X \in \mathscr{F}$, a simple counting argument shows that $(\mathscr{T}, \mathscr{F})$ is principal. Thus, (a) implies (c).

If (b) holds, the number of $R e_{i}$ in $\mathscr{T}$ must equal at least $k$ and since $R e_{k+1}, \cdots, R e_{n} \in \mathscr{F}$, it equals exactly $k$. Thus $(\mathscr{T}, \mathscr{F})$ is principal as above and so (b) imples (c).

That (c) implies (e) follows from the preceding theorem. The implications $(e) \Rightarrow(b),(e) \Rightarrow(d)$ and $(d) \Rightarrow($ a) are always valid.

We note finally that there exist splitting hereditary torsion theories over Artinian rings which are not centrally splitting. Such a torsion theory may be found for instance in the category of left modules over the ring of two by two lower triangular matrices over a field.

\section{REFERENCES}

1. J. S. Alin and E. P. Armendariz, TTF classes over perfect rings, J. Austral. Math. Soc. 11 (1970), 499-503.

2. G. Azumaya, Completely faithful modules and self-injective rings, Nagoya Math . J. 27 (1966), 697-708. MR 35 \#4253. 
3. H. Bass, Finitistic dimension and a homological generalization of semi-primary rings, Trans. Amer. Math. Soc. 95 (1960), 466-488. MR 28 \#1212.

4. R. L. Bernhardt, Splitting hereditary torsion theories over semiperfect rings, Proc. Amer. Math. Soc. 22 (1969), 681-687. MR 39 \#5639.

5. H. Cartan and S. Eilenberg, Homological algebra, Princeton Univ. Press, Princeton, N.J., 1956. MR 17, 1040.

6. S. E. Dickson, A torsion theory for Abelian categories, Trans. Amer. Math. Soc. 121 (1966), 223-235. MR 33 \#162.

7. V. Dlab, A characterization of perfect rings, Pacific J. Math. 33 (1970), 79-88. MR 41 \#6907.

8. J. P. Jans, Some aspects of torsion, Pacific J. Math. 15 (1965), 1249-1259. MR 33 \#163.

9. J. Lambek, Lectures on rings and modules, Blaisdell, Waltham, Mass., 1966. MR 34 \#5857.

10. B. L. Osofsky, A generalization of quasi-Frobenius rings, J. Algebra 4 (1966), 373-387. MR 34 \#4305.

11. Y. Utumi, Self-injective rings, J. Algebra 6 (1967), 56-64. MR 35 \#219.

Department of Mathematics, University of Kansas, Lawrence, Kansas 66044

Current address: Department of Mathematics, Virginia Polytechnic Institute and State University, Blacksburg, Virginia 24061 